\title{
Beta-blockers, hypertension, and weight gain: the farmer, the chicken, and the egg
}

\author{
Andrew J Stewart Coats *, DM, DSC \\ IRCCS, San Raffaele Pisana, Rome, Italy \\ *Corresponding author: ajscoats@aol.com \\ Hong Kong Med J 2020;26:6-7 \\ https://doi.org/10.12809/hkmj205093
}

In this issue is presented a study by KL Leung and colleagues, ${ }^{1}$ a post-hoc analysis of a dataset comprising the medication profiles of a cohort of community-dwelling older adults in Hong Kong collected during 2016, initially acquired during a study investigating the relationship between diet and the prevalence of atrial fibrillation. ${ }^{2}$ A total of 1665 Cantonese-speaking older adults (aged $\geq 65$ years) attending recreational community centres in Hong Kong were recruited, excluding any with terminal health conditions or significant cognitive impairment. The authors described an association between the use of beta-blockers in their cohort (particularly atenolol) and the prevalence of obesity, evident in the hypertensive cohort (hypertension being the likely dominant reason for prescription of beta-blockers).

It has been known for some time the use of beta-blockers is associated with mild-to-moderate weight gain, of the order of 1 to $2 \mathrm{~kg}$, although the mechanisms have not been fully established. ${ }^{3}$ This has not been investigated in an elderly community- dwelling Chinese population in the past and this publication extends our knowledge. Being retrospective it can never, of course, prove cause and effect and hence we have a chicken and egg argument. As the authors rightly point out we cannot differentiate between beta-blockers causing obesity, versus obesity leading to a preferential use of beta-blockers, which might itself be through multiple mechanisms. In addition, obese individuals have larger arms and may, by conventional blood pressure (BP) measurement (if an extra-large arm cuff is not used), lead to falsely elevated BP levels, meaning more antihypertensive medication is prescribed. I have added an allusion to a farmer to the usual chicken and egg argument in the title, for there is also the possibility that the supervising physician specifically chose beta-blockers as the class of antihypertensive agent of first choice because the patient was overweight; the authors point this out, and some guidelines have recommended betablockers for obese hypertensive patients.

Should these results concern us? As is so common in such matters, it is difficult to say for certain. One scenario is, of course, that the betablockers have been prescribed most likely in an effort to reduce cardiovascular risk, primarily due to hypertension, but in doing so they promote weight gain, which could secondarily lead to minor increases in BP, insulin resistance, the risk of diabetes and indeed cardiovascular risk itself, so that it would be self-defeating. The quantitative affect, and indeed even the causative link, are very hard to be certain about, however, but the authors are right to concern themselves with the population health consequences of these changes, even if on an individual patient basis, they seem modest differences in body weight. No other drug class was associated with changes in body mass index (BMI) with the exception of loop diuretics. The authors discount this effect saying that dry weight is not affected by loop diuretics, but of course this does not mean that the indication for the use of loop diuretics may not have affected weight, for any of heart failure, ascites, and renal impairment may cause body weight loss as well as causing the fluid retention which is the common indication for the use of loop diuretics.

What is the reason atenolol was found to be associated with an increased BMI? We cannot be certain, but the authors hypothesise a reduction in usual energy expenditure, or alterations in insulin sensitivity, but whether the weight gain is the cause or consequence of the latter is uncertain. The authors conclude that beta-blockers should be avoided in the first-line treatment of hypertension because of this association with obesity, yet this conclusion is not soundly based on the data presented, where a nearly similar number of patients were taking metoprolol as atenolol with no difference in obesity rates. If there were to be any conclusion then it would have to be restricted to atenolol and not all beta-blockers. There are other reasons not to use atenolol such as its relatively short half-life and hence poor 24-hour BP control, and lack of trial data to show beneficial effects on major outcomes, other than in the postmyocardial infarction setting. Newer vasodilating beta-blockers, such as carvedilol and nebivolol, may well have very different risk-benefit relationships in hypertension if they had been studied in major hypertension trials, and they may for all we know, not have the same effect on BMI as atenolol, so there still remains much to explore for a fuller understanding of the true role of modern beta-blockers in the 
management of hypertension. There is also an alternative explanation for some of these results, and that may be that some of the weight gain may be in fact be beneficial, as it is only at population level and not prospective individual changes in weight that we see.

It can be seen that, in addition to a higher percentage of the studied cohort with obesity receiving a beta-blocker, there is also a lower percentage of people who are underweight, so there may simply have been a population shift in weight, and who can say for certain this is due to weight gain, and not due to an element at least, of a lower rate of weight loss in some of the studied population. Whilst it is true in middle age, that the population on average increases in weight, in the older age range, there is a progressive decline in weight, which can become quite severe and be associated with frailty and loss of skeletal muscle mass and function, termed sarcopaenia. ${ }^{4}$ In the related field of cachexia research there has been interest in the use of betablockers to therapeutically prevent severe muscle loss and weight loss because of the anti-catabolic effect of beta-1 receptor antagonism, and at least for some beta-blockers also the potential for some muscle anabolic partial agonism of the beta- 2 receptor. ${ }^{5}$ Whilst both atenolol and metoprolol, the two most common beta-blockers seen to be taken by the patients in this study are predominately beta- 1 selective and without beta- 2 agonist effects, subtle differences in the receptor specificities may explain why, in particular, atenolol was associated with a higher body weight than metoprolol. But with relatively small numbers and the retrospective nature of the study we cannot explore this any further. Thus, I congratulate the authors for their work and feel it adds to our understanding, but I caution against assuming we fully understand the significance of the use of beta-blockers in health and disease, particularly in older adults.

\section{Author contributions}

The author had full access to the data, contributed to the study, approved the final version for publication, and take responsibility for its accuracy and integrity. The author contributed to concept or design, analysis or interpretation of data, drafting of the article, and critical revision for important intellectual content.

\section{Conflicts of interest}

The author declares no conflicts related to this work. Outside of this work, in the last 3 years, the author declares having received honoraria and/or lecture fees from: AstraZeneca, Bayer, Menarini, Novartis, Nutricia, Servier, Vifor, Actimed, Cardiac Dimensions, CVRx, Enopace, Faraday, Gore, Impulse Dynamics, Respicardia, Stealth Peptides, V-Wave, Corvia, Arena, and ESN Cleer.

\section{Funding/support}

This editorial received no specific grant from any funding agency in the public, commercial, or not-for-profit sectors.

\section{References}

1. Leung KL, Fong W, Freedman B, Bajorek B, Lee VW. Association between beta-blocker use and obesity in Hong Kong Chinese elders: a post-hoc analysis. Hong Kong Med J 2020;26:27-34.

2. Leung K, Fong W, Lau PS, et al. Impact of dining out and food intake pattern on atrial fibrillation prevalence in Hong Kong Chinese elders. Value Health 2018;21:S57.

3. Davis BR, Oberman A, Blaufox MD, et al. Effect of antihypertensive therapy on weight loss. The Trial of Antihypertensive Interventions and Management Research Group. Hypertension 1992;19:393-9.

4. Tieland M, Trouwborst I, Clark BC. Skeletal muscle performance and ageing. J Cachexia Sarcopenia Muscle 2018;9:3-19.

5. Stewart Coats AJ, Ho GF, Prabhash K, et al. Espindolol for the treatment and prevention of cachexia in patients with stage III/IV non-small cell lung cancer or colorectal cancer: a randomized, double-blind, placebo-controlled, international multicentre phase II study (the ACT-ONE trial). J Cachexia Sarcopenia Muscle 2016;7:355-65. 